\title{
(-)-Epigallocatechin-3-gallate suppresses hepatic preneoplastic lesions developed in a novel rat model of non-alcoholic steatohepatitis
}

Takafumi Sumi ${ }^{1}$, Yohei Shirakami ${ }^{1}$, Masahito Shimizu ${ }^{1,3^{*}}$, Takahiro Kochi ${ }^{1}$, Tomohiko Ohno $^{1}$, Masaya Kubota ${ }^{1}$, Makoto Shiraki ${ }^{1}$, Hisashi Tsurumi ${ }^{1}$, Takuji Tanaka ${ }^{2}$ and Hisataka Moriwaki ${ }^{1}$

\begin{abstract}
Purpose: Non-alcoholic fatty liver disease (NAFLD) ranges from simple steatosis to non-alcoholic steatohepatitis (NASH). NASH, which is accompanied by increased oxidative stress and inflammation in the liver, is associated with hepatic carcinogenesis. Green tea catechins (GTCs) possess anti-oxidant, anti-inflammatory, and cancer-preventive properties. In this study, we investigated whether (-)-epigallocatechin-3-gallate (EGCG), a major component of GTCs, inhibits NAFLD/NASH-related liver tumorigenesis.

Methods: Male 8-week-old Sprague-Dawley (SD) rats were administered a single intraperitoneal injection of a hepatic carcinogen diethylnitrosamine (DEN, $30 \mathrm{mg} / \mathrm{kg}$ body weight) and then fed a high-fat diet (HFD) for 7 weeks. The rats were also provided tap water containing $0.01 \%$ or $0.1 \%$ EGCG during the experiment.

Results: At sacrifice, the livers of SD rats treated with DEN and HFD exhibited marked development of glutathione S-transferase placental form (GST-P)-positive foci, a hepatic preneoplastic lesion, and this was associated with hepatic steatosis, oxidative stress and inflammation, and hepatocyte proliferation. EGCG administration, however, inhibited the development of GST-P-positive foci by decreasing hepatic triglyceride content, reducing hepatic fibrosis, lowering oxidative stress, attenuating inflammation, and inhibiting excessive hepatocyte proliferation in DEN- and HFD-treated SD rats. These findings suggest that the experimental model of SD rats treated with HFD and DEN, in which histopathological and pathophysiological characteristics of NASH and the development of hepatic premalignant lesions were observed, might facilitate the evaluation of liver tumorigenesis associated with NAFLD/NASH.
\end{abstract}

Conclusions: Administering EGCG, a GTC, might serve as an effective chemoprevention modality for NAFLD/NASH-related liver tumorigenesis.

Keywords: Non-alcoholic steatohepatitis; Liver tumorigenesis; Oxidative stress; Inflammation; EGCG

\section{Background}

Non-alcoholic fatty liver disease (NAFLD), which is considered a hepatic manifestation of the metabolic syndrome, is currently one of the most common chronic liver diseases worldwide. NAFLD is classified into simple steatosis and non-alcoholic steatohepatitis (NASH), the latter being a severe condition of inflamed fatty liver

\footnotetext{
* Correspondence: shimim-gif@umin.ac.jp

${ }^{1}$ Department of Internal Medicine, Gifu University Graduate School of Medicine, Gifu, Japan

${ }^{3}$ Department of Internal Medicine/Gastroenterology, Gifu University Graduate School of Medicine, 1-1 Yanagido, Gifu 501-1194, Japan

Full list of author information is available at the end of the article
}

that can, in time, lead to hepatic fibrosis, cirrhosis, hepatocellular carcinoma (HCC) development, and result in increased mortality (Chiang et al., 2011; Cusi, 2012; Siegel and Zhu, 2009). Steatohepatitis has been indicated by epidemiological studies to be a significant risk factor for developing HCC, at an annual HCC incidence of $2 \%-3 \%$ in patients with NASH (Adams et al., 2005; Ascha et al., 2010). In 1998, Day and James proposed a "two-hit theory" to explain NAFLD/NASH pathogenesis: the first hit, hepatic steatosis, increases the sensitivity of the liver to proinflammatory insults, while the second hit involves oxidative stress (Day and James, 1998). Oxidative 
stress, which is associated with HCC development (Suzuki et al., 2013), is substantially higher in NASH patients than in NAFLD patients and normal control subjects (Sanyal et al., 2001). Moreover, tumor necrosis factor (TNF)- $\alpha$ and interleukin (IL)-6, both of which are major proinflammatory cytokines, play a critical role in obesity-related steatohepatitis and subsequent liver tumorigenesis (Park et al., 2010).

Several animal models that mimic the pathophysiological mechanisms associated with NAFLD/NASH-related liver carcinogenesis have been developed recently (Hebbard and George, 2011; Schattenberg and Galle, 2010; Kochi et al., 2013). For instance, $d b / d b$ mice, which exhibit obesity, diabetes, dyslipidemia, and severe steatosis, are susceptible to liver tumorigenesis induced by a hepatic carcinogen diethylnitrosamine (DEN), and thus are regarded as useful animal models for investigating pathobiology of NAFLD/NASH-related liver carcinogenesis and for screening the chemopreventive effects of various compounds either synthetic or natural on NAFLD/ NASH-related liver carcinogenesis (Iwasa et al., 2010; Shimizu et al. 2011a, 2011b, 2011c). Recently, Wang et al. (Wang et al. 2009) reported that NASH induced by a high-fat diet (HFD) promoted DEN-initiated early hepatocarcinogenesis in Sprague-Dawley (SD) rats and that this was associated with increased oxidative stress and inflammation. This animal model is also useful for investigating the efficacy of certain types of synthetic compounds and/or phytochemicals in preventing NASHpromoted liver carcinogenesis (Wang et al., 2010).

The prevalence of NAFLD/NASH has risen recently in parallel with the dramatic increase in obesity and its related metabolic abnormalities, especially diabetes mellitus (Chiang et al., 2011; Cusi, 2012; Siegel and Zhu, 2009), indicating an urgent requirement for developing an effective strategy to treat NAFLD/NASH and, consequently, to prevent NAFLD/NASH-related liver carcinogenesis. A recent randomized trial (Sanyal et al., 2010) has shown that treatment with vitamin $\mathrm{E}$, an antioxidant, reduces steatosis and lobular inflammation in the liver of NASH patients. Reducing oxidative stress and inhibiting the inflammation induced by obesity and steatosis are also effective in preventing obesity- and diabetes-related hepatotumorigenesis (Shimizu et al. 2013, 2012). These reports suggest that targeting oxidative stress and chronic inflammation is an optimal strategy for preventing NAFLD/NASH-related liver carcinogenesis.

Green tea catechins (GTCs) might be one of the most promising candidate compounds for preventing NAFLD/ NASH-related liver carcinogenesis because they are considered to protect against metabolic disorders such as NAFLD (Masterjohn and Bruno, 2012; Thielecke and Boschmann, 2009) and also display cancer chemopreventive properties in various tissues, including the liver (Shimizu et al. 2011b; Kochi et al., 2013; Yang et al., 2009). In this study, we developed a novel rat model of NAFLD/NASH-related carcinogenesis and investigated the potential capacity of (-)-epigallocatechin-3-gallate (EGCG), a major component of GTCs, to inhibit the occurrence of HFD- and DEN-induced glutathione $S$ transferase placental form (GST-P)-positive foci, an indicator of preneoplastic HCC lesions in rats (Tsuda et al., 2003; Ando et al., 2007).

\section{Results}

\section{General observations}

At the end of the experimental period, the rats in the 3 groups exhibited no significant differences with respect to the mean body, liver, and kidney weights (Table 1). Histopathological examination revealed that administering EGCG produced no detectable toxic effects on critical organs including the liver, kidney, and spleen (data not shown).

\section{Effects of EGCG on hepatic steatosis and serum ALT levels in rats}

At sacrifice, macrovesicular steatosis with ballooned hepatocytes, Mallory-Denk bodies, and foci of inflammatory cells, which are a recognized feature of NASH (Kleiner et al., 2005), were observed in the livers of rats in all groups, indicating that the histopathological characteristics that develop in this animal model reproduce those of NASH. However, these pathological effects were alleviated by the administration of $0.01 \%$ and $0.1 \%$ EGCG (Figure 1a and 1b). In particular the total NAFLD activity score (NAS) consisting of the steatosis, inflammation, and ballooning scores, was significantly lower in 0.1\% EGCG-treated rats than in EGCG-untreated control rats (Figure 1c, $P<0.05$ ). Similar results were obtained following measurement of intrahepatic lipid content: the levels of liver triglycerides in

Table 1 Body and organ weights of the experimental rats

\begin{tabular}{lcccc}
\hline Group No. & EGCG & No. of rats & $\begin{array}{c}\text { Body } \\
\text { weight (g) }\end{array}$ & \multicolumn{2}{c}{ Relative organ weight (g/100 g body weight) } \\
\hline 1 & - & 7 & $512.7 \pm 37.8^{\text {a }}$ & Kidneys \\
2 & $0.01 \%$ & 6 & $495.5 \pm 29.0$ & $3.8 \pm 0.5$ \\
3 & $0.1 \%$ & 6 & $501.6 \pm 44.3$ & $3.9 \pm 0.6$ \\
\hline
\end{tabular}



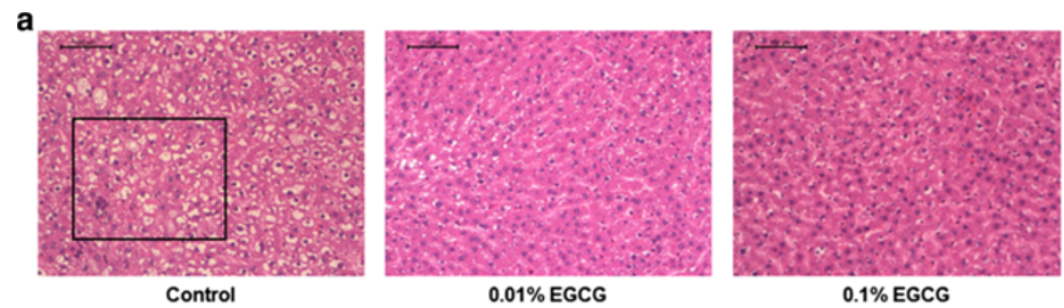

b

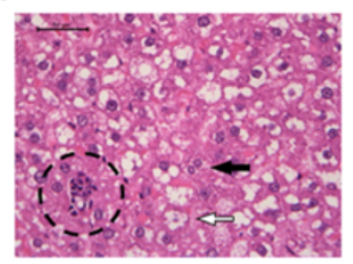

。

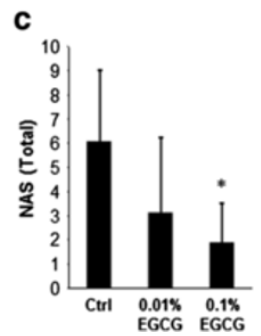

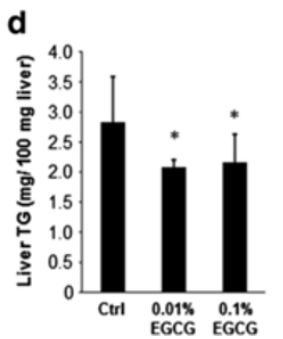

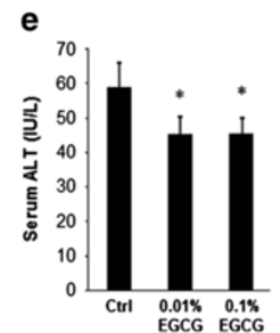

Figure 1 Hepatic histopathology, intrahepatic levels of triglycerides, and serum levels of ALT. (a) and (b) H\&E staining of liver sections from the experimental rats. (a) Representative photomicrographs of liver sections of rats treated DEN/HFD with or without EGCG. Bars, $100 \mu \mathrm{m}$. (b) An enlarged photo of the liver section from a rat treated DEN/HFD without EGCG (an enclosed area with square in Figure 1a). Note ballooned hepatocytes (indicated by the white arrow), Mallory-Denk bodies (indicated by the black arrow), and focus of inflammatory cells (circled by dotted line). Bars, 50 mm. (c) The NAS was determined from histopathological analysis. (d) Hepatic lipids were extracted from frozen liver samples of rats in the 3 groups, and the triglyceride (TG) levels were measured. (e) Blood samples were collected at sacrifice and the serum levels of alanine aminotransferase (ALT) were determined. The values are expressed as mean \pm SD. ${ }^{*} P<0.05$. Ctrl, control group.

the DEN- and HFD-treated SD rats were significantly decreased when they received $0.01 \%$ and $0.1 \%$ EGCG instead of water (Figure 1d, $P<0.05$ ). The serum alanine aminotransferase (ALT) levels were also significantly decreased with EGCG treatment at both concentrations relative to the levels in the water-treated group (Figure 1e, $P<0.05$ ), indicating that EGCG protected against hepatic steatosis and subsequent hepatocyte injury induced by DEN and HFD.

\section{Effects of EGCG on liver fibrosis in rats}

Examination of Azan-stained liver sections revealed that DEN- and HFD-treated SD rats developed perisinusoidal fibrosis, but that EGCG administration reduced liver fibrosis in the animals (Figure 2a). The liver fibrosis score was also significantly decreased by EGCG administration (Figure 2b). Furthermore, EGCG treatment significantly lowered (relative to control) the hepatic levels of TIMP-1 and TIMP-2 mRNA in the DEN- and HFD-treated SD rats (Figure 2c, $P<0.05$ ).

\section{Effects of EGCG on the development of hepatic preneoplastic lesions in rats}

At the end of the experiment, GST-P-positive foci were detected in the livers of the rats, all of which had received DEN/HFD (Figure 3a). However, compared with rats in the control group that were not treated with EGCG, rats treated with EGCG showed a significant reduction in the number of GST-P-positive foci: $86 \%$ and $87 \%$ reduction relative to control following treatment with $0.01 \%$ and $0.1 \%$ EGCG, respectively (Figure $3 \mathrm{~b}, P<0.01$ ).

\section{Effects of EGCG on oxidative stress in rats}

Oxidative stress plays a critical role in the NAFLD-toNASH progression and HCC development (Chiang et al., 2011; Cusi, 2012; Siegel and Zhu, 2009). Therefore, we examined the levels of oxidative stress and antioxidant biomarkers in the experimental rats. Rats administrated with $0.1 \%$ EGCG had significantly reduced levels of urinary 8-OHdG, a marker of DNA damage induced by oxidative stress, compared with EGCG-untreated control rats (Figure $4 \mathrm{a}, P<0.01$ ). The serum d-ROM level, which reflects serum hydroperoxide levels, was also decreased relative to control in rats treated with $0.01 \%$ and $0.1 \%$ EGCG (Figure 4b, $P<0.01$ ). Moreover, the antioxidant enzyme (catalase and GPx-1) levels were significantly increased in the livers of rats that received EGCG treatment (Figure 4c, $P<0.05$ ). These results indicate that drinking EGCG attenuated both systemic and hepatic oxidative stress in our rat model of NAFLD/ NASH-related liver tumorigenesis.

\section{Effects of EGCG on hepatic expression of TNF-a, IL-6, and} $\mathrm{IL}-1 \beta$ mRNA in rats

Chronic inflammation is implicated in the progression of $\mathrm{NASH}$ and subsequent liver carcinogenesis (Chiang et al., 2011; Cusi, 2012; Siegel and Zhu, 2009; Park et al., 2010). Therefore, the mRNA expression levels of 3 inflammatory 
a

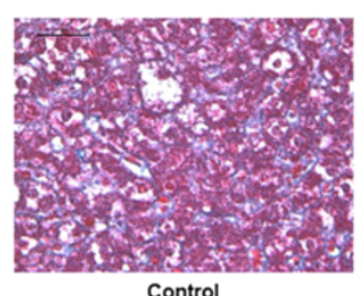

b

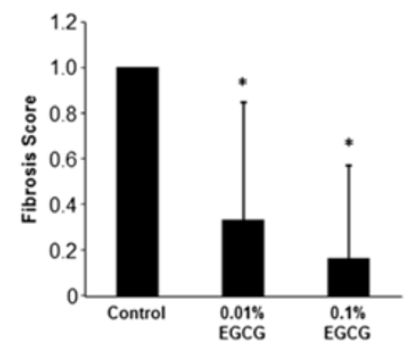

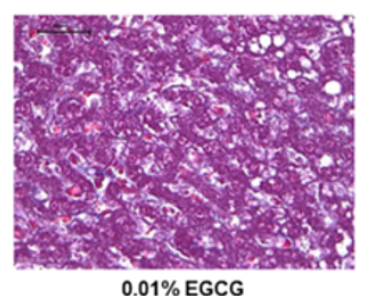

$0.01 \%$ EGCG

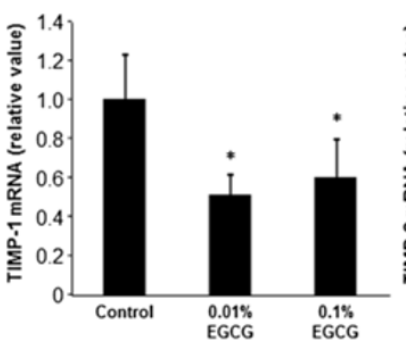

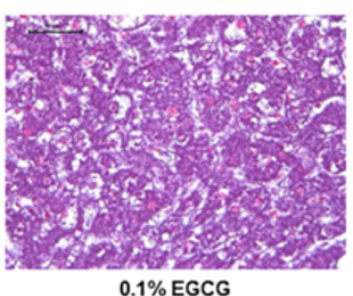

$0.1 \%$ EGCG

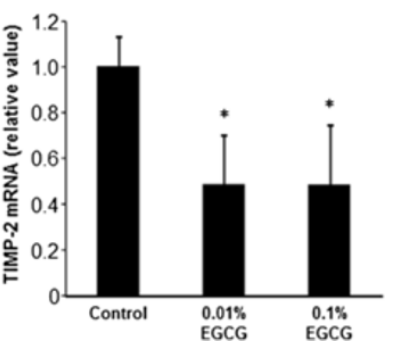

Figure 2 Liver fibrosis and hepatic expression levels of TIMP-1 and TIMP-2 mRNA. (a) Representative photomicrographs of Azan staining of liver tissues to show fibrosis. Bars, $50 \mu \mathrm{m}$. Note reduction of liver fibrosis (blue) by the treatment with EGCG. (b) Liver fibrosis evaluation was based on the NAS system. (c) After total RNA was isolated from the livers of the rats, the levels of TIMP-1 and TIMP-2 mRNA were measured using specific primers and quantitative real-time RT-PCR. The values are expressed as mean \pm SD. * $P<0.05$.

mediators, TNF- $\alpha$, IL-6, and IL-1 $\beta$, were measured in the livers of DEN- and HFD-treated SD rats. As shown in Figure 5, quantitative real-time RT-PCR analysis revealed that rats that received 0.1\% EGCG exhibited significantly lower hepatic levels of TNF- $\alpha(P<0.05)$, IL-6 $(P<0.01)$, and IL-1 $\beta(P<0.05)$ mRNA than control rats that received only water, and the hepatic levels of IL- 6 mRNA were also decreased by the administration of a low dose $(0.01 \%)$ of EGCG $(P<0.01)$.
Effects of EGCG on hepatocyte proliferation and hepatic expression of cyclin D1 mRNA in rats

The PCNA-labeling index of non-lesional hepatocytes in DEN- and HFD-treated SD rats was determined based on the findings of immunohistochemical examination of sections (Figure 6a). The mean PCNA-labeling indices measured for rats administered $0.01 \%$ and $0.1 \%$ EGCG were significantly lower than that for EGCG-untreated control rats (Figure $6 \mathrm{~b}, P<0.01$ ). Furthermore, the levels

a

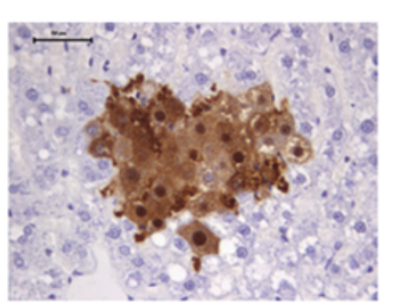

Control

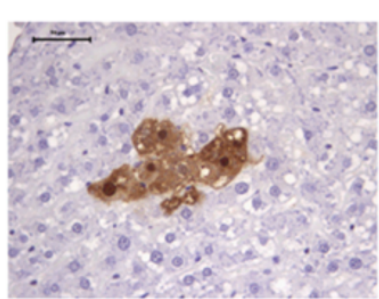

$0.01 \%$ EGCG

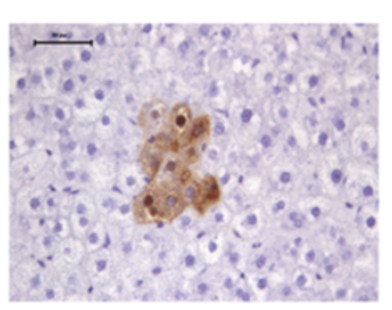

$0.1 \%$ EGCG

b

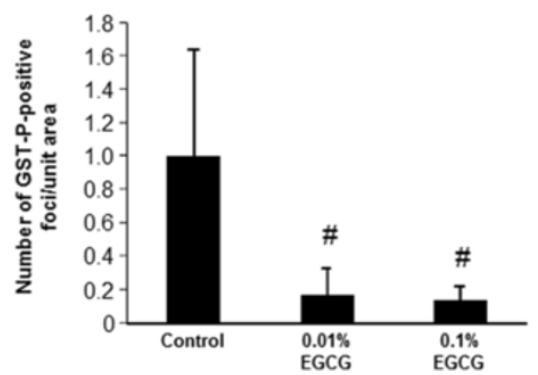

Figure 3 The development of GST-P-positive foci in the livers. (a) Representative photomicrographs of GST-P-positive foci. Note a large GST-P focus in a rat treated with DEN/HFD. Bars, $50 \mu \mathrm{m}$. (b) The average numbers of GST-P-positive foci formed in the livers of the rats in the 3 groups. The values are expressed as mean \pm SD. \# $P<0.01$. 

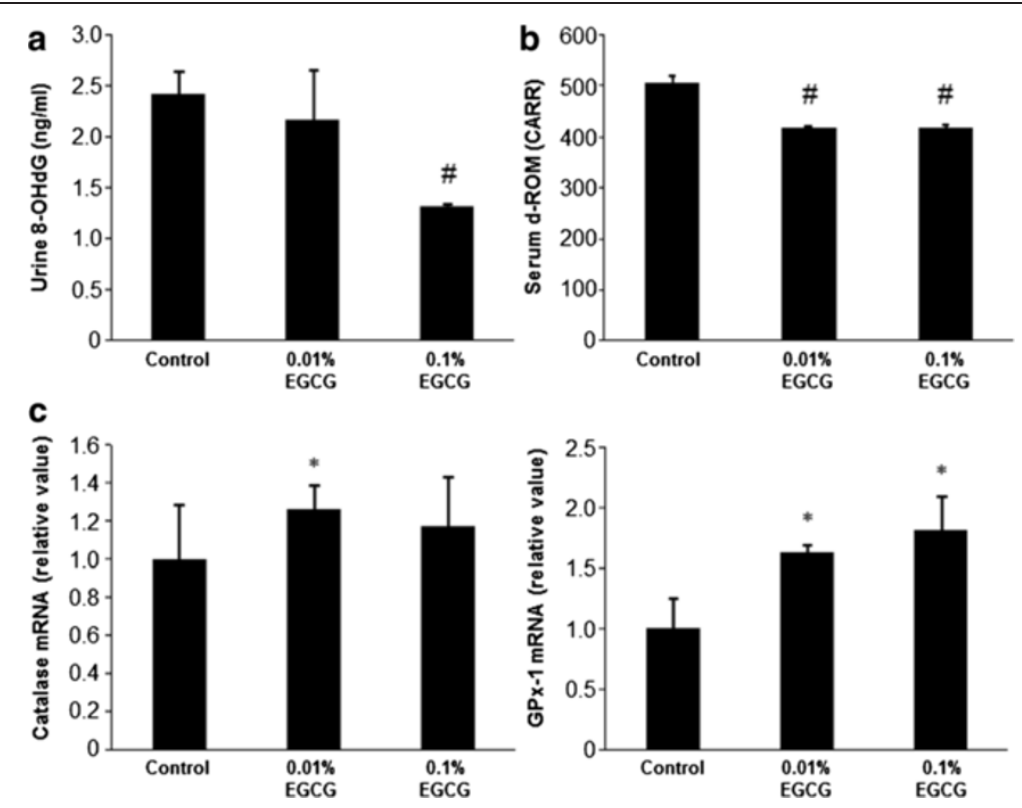

Figure 4 Urinary 8-OHdG and serum d-ROM levels and on the hepatic catalase and GPx-1 mRNA levels. (a) The urinary 8-OHdG levels were measured using ELISA. (b) Hydroperoxide levels in the serum were determined using the d-ROM test. (c) Levels of catalase and GPX-1 mRNA in the livers of the experimental rats were measured using specific primers and quantitative real-time RT-PCR. The values are expressed as mean \pm SD. $\# P<0.01,{ }^{*} P<0.05$.

of cyclin D1 mRNA in liver were also markedly decreased in EGCG-treated rats relative to that in control rats (Figure 6c, $P<0.05$ ), indicating that EGCG significantly inhibited hepatocyte proliferation in DEN- and HFD-treated SD rats.

\section{Discussion}

An increase in the prevalence of NAFLD/NASH, which can progress to $\mathrm{HCC}$, is a major healthcare problem worldwide (Chiang et al., 2011; Cusi, 2012; Siegel and Zhu, 2009). Therefore, developing an effective strategy for preventing NAFLD/NASH-related liver tumorigenesis is critical for improving the prognosis of patients with these diseases. The results of this study clearly indicate that EGCG, a GTC, effectively prevents the development of hepatic preneoplastic lesions, which manifest as GST-
P-positive foci, in our rat model of NAFLD/NASH-related liver tumorigenesis. The rodent model used in this study, which was modified from a model described previously (Wang et al., 2009), reflects the pathological alterations implicated in NAFLD/NASH and NAFLD/NASH-related liver tumorigenesis, including the induction of oxidative stress and chronic inflammation (Chiang et al., 2011; Cusi, 2012; Siegel and Zhu, 2009; Park et al., 2010). Therefore, we consider the present model to be an appropriate and a useful animal model for analyzing the mechanisms of NAFLD/NASH-related liver tumorigenesis and for evaluating the efficacy of specific chemopreventive agents that can suppress such tumorigenesis.

Among the numerous pathophysiological conditions associated with NAFLD/NASH, oxidative stress is regarded as one of the key mechanisms for the development of
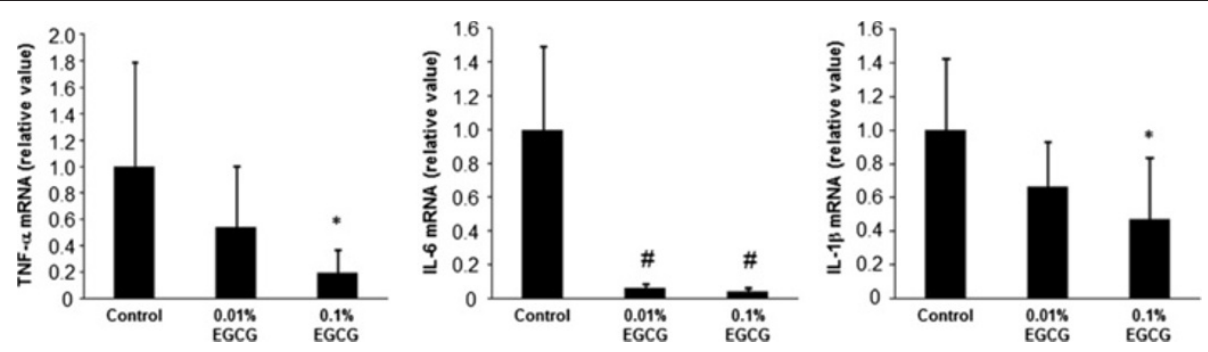

Figure 5 Hepatic expression of TNF- $\boldsymbol{\alpha}, \mathrm{IL}-\mathbf{6}$, and IL-1 $\beta$ mRNA. Total RNA was isolated from the livers of rats in the 3 groups and the expression levels of TNF- $a, \mathrm{IL}-6$, and IL-1 $\beta$ mRNA were determined using specific primers and quantitative real-time RT-PCR. The values are expressed as mean $\pm \mathrm{SD}$. \# $P<0.01,{ }^{*} P<0.05$. 
a

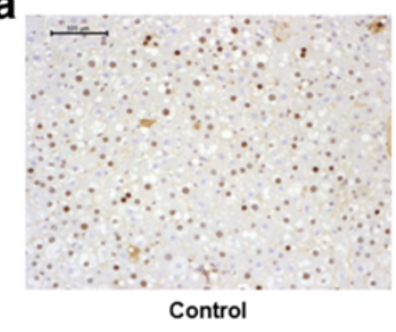

b

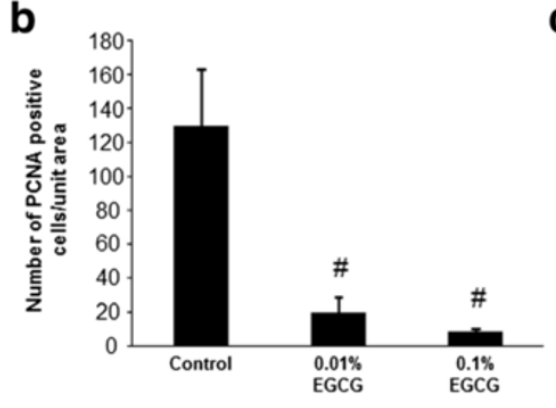

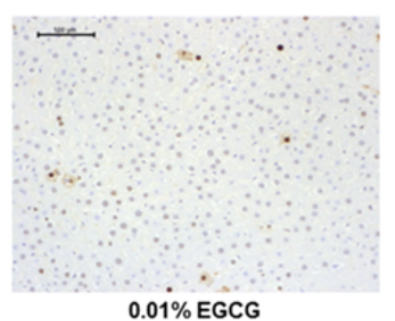

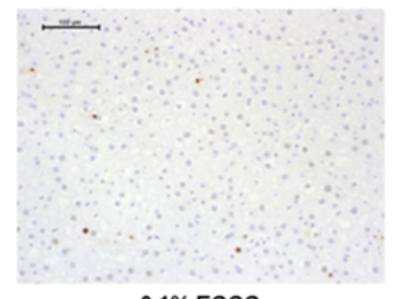

$0.1 \%$ EGCG

C

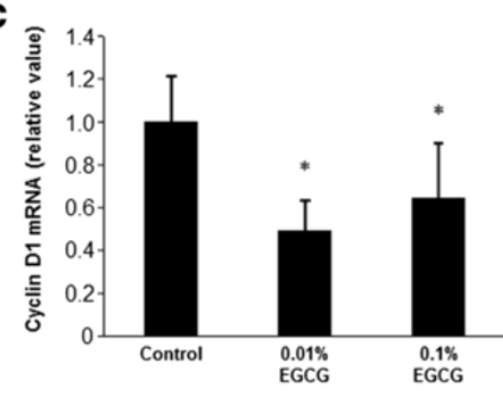

Figure 6 Hepatocyte proliferation and hepatic expression of cyclin D1 mRNA. (a) Immunohistochemical labeling for PCNA in the livers of the experimental rats. Bars, $100 \mu \mathrm{m}$. (b) The PCNA-labeling index in non-lesional hepatocytes was determined by counting the hepatocytes with PCNA-positive nuclei to calculate their percentage in the hepatocyte population. (c) The cyclin D1 mRNA levels in the liver of the experimental rats were determined using specific primers and quantitative real-time RT-PCR. The values are expressed as mean $\pm \mathrm{SD}$. \# $P<0.01,{ }^{*} P<0.05$.

HCC. In the progression of NAFLD to NASH, an increase in oxidative stress, which is defined as the overproduction of reactive oxygen species combined with inadequate antioxidative defense mechanisms, produces DNA damage and gene mutations associated with liver carcinogenesis (Chiang et al., 2011; Cusi, 2012; Siegel and Zhu, 2009; Park et al., 2010). Conversely, treatment with antioxidants such as vitamin E can reduce hepatic steatosis, lobular inflammation, and serum ALT levels, as shown by a clinical trial conducted in NASH patients (Sanyal et al., 2010). Administering pentoxifylline, which is known to decrease oxidative stress and inhibit TNF- $\alpha$ expression, also improved the histological features of NASH in a recent randomized placebo-controlled trial (Zein et al., 2011). In this study, treatment with EGCG lowered the levels of oxidative stress-associated markers such as urinary 8-OHdG and serum d-ROM, whereas elevated the mRNA levels of two antioxidant enzymes, catalase and GPx-1, in the liver of DEN- and HFD-treated SD rats. These findings suggest that EGCG suppressed NAFLD/NASH-related liver tumorigenesis at least partly by reducing systemic and hepatic oxidative stress. Our results are consistent with those of previous studies showing that GTCs can protect against both oxidative stress and the progression of NAFLD to NASH (Park et al., 2011; Ueno et al., 2009; Kuzu et al., 2008).

Besides oxidative stress, chronic inflammation is critically involved in NAFLD/NASH-related liver tumorigenesis (Chiang et al., 2011; Cusi 2012; Siegel and Zhu, 2009; Park et al., 2010). Among the proinflammatory cytokines related to the progression of NASH, TNF- $\alpha$ and IL- 6 play a pivotal role in hepatocyte injury and inflammation, which increase HCC risk (Park et al., 2010). Therefore, targeting TNF- $\alpha$ and IL- 6 might be an effective method to suppress NAFLD/NASH-related liver tumorigenesis. GTCs are widely recognized to exert cancer-preventive effects partly by inhibiting the expression of TNF- $\alpha$ and IL-6 (Shimizu et al. 2011b; Kochi et al., 2013; Shirakami et al. 2008), indicating that the suppression of inflammation is one of the key mechanisms by which GTCs prevent cancer development. In this study, we found that mRNAs encoding TNF- $\alpha$, IL- 6 , and IL-1 $\beta$ were expressed at significantly lower levels in the livers of EGCG-treated rats compared to that in EGCG-untreated rats. Therefore, in agreement with previous reports (Shimizu et al. 2011b; Kochi et al., 2013; Shirakami et al. 2008), the results of this study suggest that EGCG consumption suppressed the development of GST-P-positive foci in DEN- and HFD-treated SD rats by attenuating chronic inflammation.

In addition to reducing oxidative stress and chronic inflammation, both of which are secondary manifestations of NASH, administering EGCG improved hepatic steatosis, a primary manifestation of NASH (Day and James, 1998), decreased serum ALT levels, ameliorated liver fibrosis, and inhibited excessive hepatocyte proliferation in this study. GTCs have been demonstrated to attenuate hepatic fat accumulation in several laboratory animal studies (Kuzu et al., 2008; Kochi et al., 2013; Shimizu et al. 2011b; Park et al., 2011; Ueno et al., 2009); 
these reports combined with the findings of this study are of interest because hepatic steatosis per se can induce hepatocyte proliferation and hepatic hyperplasia, both of which initiate the hepatic neoplastic process by increasing hepatocyte proliferative activity (Yang et al., 2001). Moreover, hepatic steatosis is critically related to liver fibrosis, which is a strong risk factor for the development of HCC (Powell et al., 2005). Therefore, suppression of hepatic steatosis and fibrosis by EGCG treatment might help to inhibit the progression of NAFLD/NASH-related liver tumorigenesis at an early stage.

Because GST-P-positive foci are generally accepted to be precursors or preneoplastic lesions of $\mathrm{HCC}$ in rats (Tsuda et al., 2003; Ando et al., 2007), the rodent model used in this study appears to be well suited for screening reagents that can prevent NAFLD/NASH-related liver tumorigenesis. However, the current study has one limitation that hepatocellular neoplasms, including HCC, did not develop within the experimental period. Because the duration of the experiment (7 weeks) might have been insufficient for the development of hepatic neoplasms, future studies should be conducted using longer-term experiments to confirm that HFD- and DEN-treated SD rats develop hepatocellular neoplasms.

Finally, we emphasize again that targeting metabolic abnormalities, especially oxidative stress and chronic inflammation, might be one of the most practical approaches for treating NAFLD/NASH and preventing NAFLD/NASH-related liver carcinogenesis (Shimizu et al. 2013, 2012). We consider GTCs including EGCG to be potentially effective and key candidates for this purpose, because these agents can target metabolic abnormalities and thus prevent relevant tumorigenesis, as shown by the results of this study and those from previous reports (Shimizu et al. 2011b; Kochi et al., 2013; Shimizu et al. 2008b; Thielecke and Boschmann, 2009; Grove and Lambert, 2010). Recent clinical trials have also demonstrated that GTC supplementation potently prevents the development of both colorectal adenomas and prostate cancers without causing adverse effects (Shimizu et al. 2008a; Bettuzzi et al., 2006). These beneficial effects of GTCs reported in clinical trials strongly encourage the clinical usage of GTCs for treating NAFLD/ $\mathrm{NASH}$ patients to prevent the metabolic abnormalities, such as steatosis, hyperlipidemia, and hyperinsulinemia, as well as liver carcinogenesis.

\section{Conclusions}

In conclusion, administering EGCG effectively suppresses the early stage of hepatocarcinogenesis in our rat model of NAFLD/NASH by attenuating oxidative stress and chronic inflammation. Application of GTCs represents a potential new strategy for preventing the development of hepatic neoplasms in NAFLD/NASH patients.

\section{Methods}

Animals and chemicals

Male 7-week-old SD rats were obtained from Japan SLC, Inc. (Shizuoka, Japan) and humanely maintained at Gifu University Life Science Research Center in accordance with the Institutional Animal Care Guidelines. DEN was purchased from Sigma-Aldrich Co. LLC. (St. Louis, MO, USA). HFD-60 (HFD, $506.2 \mathrm{kcal} / 100 \mathrm{~g}$ ) with $62.2 \%$ of the calories derived from fat was purchased from Oriental Yeast (Tokyo, Japan) (Table 2). EGCG was obtained from Mitsui Norin Co. Ltd. (Tokyo, Japan).

\section{Experimental procedure}

The present study was approved by the Experimental Animal Research Committee of Gifu University. After 1 -week-acclimatization with regular chow, all rats $(n=19)$ received a single intraperitoneal injection of DEN $(30 \mathrm{mg} / \mathrm{kg}$ body weight) and were then randomly divided into 3 groups. Following DEN injection, the rats in Groups 2 and 3 ( $\mathrm{n}=6$ in both groups) were provided tap water containing $0.01 \%$ or $0.1 \%$ EGCG, respectively, whereas the rats in Group $1(n=7)$ were provided tap water throughout the experiment, which lasted 7 weeks. All rats were fed a pelleted HFD throughout the experiment after DEN injection. At the end of the experiment, the 15-week-old rats were sacrificed by $\mathrm{CO}_{2}$ asphyxiation and the development of GST-P-positive foci was evaluated. The concentration of EGCG used (0.1\%), which was established based on the findings of previous chemopreventive studies

Table 2 Composition and calories of the experimental diet HFD-60

\begin{tabular}{lc}
\hline Ingredients & (g/kg diet) \\
\hline Casein & 256.0 \\
Corn starch & 160.0 \\
Sucrose & 55.0 \\
Dextrose & 60.0 \\
Cellulose & 66.1 \\
Soybean oil & 20.0 \\
Lard & 330.0 \\
Vitamin mixture & 35.0 \\
Mineral mixture & 10.0 \\
Calcium carbonate & 1.8 \\
L-cysteine & 3.6 \\
Choline bitartrate & 2.5 \\
Energy & (kcal/kg) \\
& 5062 \\
Protein & $\mathbf{( \% )}$ \\
Fat & 18.2 \\
Carbohydrate & 62.2 \\
\hline
\end{tabular}


(Shimizu et al. 2011b; Kochi et al., 2013; Shirakami et al., 2008; 2009), was, in terms of units per body weight, within the physiological range measured in humans after daily intake of GTCs (Wang et al., 1991). Previously, GST-P-positive foci were markedly induced by DEN injection in HFD fed rats, but not in rats fed a normal diet (Wang et al. 2009), therefore we did not use a control group that was fed normal chow diet after DEN injection in the present study.

\section{Histopathological examination and immunohistochemical} analyses for GST-P and proliferating cell nuclear antigen (PCNA)

Maximum sagittal sections of 3 liver sublobes (central, lateral, and right-anterior) were used for histopathological examination. Formalin-fixed and paraffin-embedded livers were stained with hematoxylin \& eosin (H\&E) for conventional histopathology or with Azan stain to detect liver fibrosis. The histological features of the livers were evaluated using the NAFLD activity score (NAS) system (Kleiner et al., 2005), and the development of liver fibrosis was determined as described previously (Kleiner et al., 2005). Immunohistochemistry for GST-P (Ando et al., 2007) and PCNA (Iwasa et al., 2010) was performed using primary antibodies against GST-P (MBL Co. Ltd., Nagoya, Japan) and PCNA (Santa Cruz Biotechnology, Inc., Santa Cruz, CA, USA), respectively. The number of GST-P-positive foci, which was set as 3 or more positive cells (Kochi et al., 2013), was assessed per unit area (per $\mathrm{cm}^{2}$ ). In the PCNA-immunostained sections, cells with intensely stained nuclei were considered to be positive for PCNA, and the indices (\% PCNA-positive) were determined by counting at least 500 hepatocytes in each section (total of 3000 hepatocytes per rat) (Iwasa et al., 2010).

RNA extraction and quantitative real-time RT-PCR analysis Total RNA was isolated from the livers of the rats by using RNeasy Mini kit (QIAGEN, Venlo, Netherlands) with on-column DNase I-digestion (Terakura et al., 2012). From $0.2 \mu \mathrm{g}$ of total RNA, cDNAs were amplified using High-Capacity cDNA Reverse Transcription Kit (Applied Biosystems, Santa Clara, CA, USA) and an automated thermal cycler (Bio-Rad Laboratories, Hercules, CA, USA). Quantitative real-time reverse transcription-PCR (RT-PCR) analysis was performed using specific primers that amplify the TNF- $\alpha$, IL-1 $\beta$, IL- 6 , tissue inhibitor of metalloproteinases (TIMP)-1, TIMP-2, glutathione peroxidase (GPx)-1, catalase, cyclin D1, and glyceraldehyde-3phosphate dehydrogenase (GAPDH) genes. The sequences of these primers, which were obtained using PrimerBLAST (http://www.ncbi.nlm.nih.gov/tools/primer-blast/), are shown in Table 3. Each sample was analyzed on a LightCycler Nano (Roche Diagnostics, Mannheim,
Table 3 Primer sequences

\begin{tabular}{|c|c|c|}
\hline Target gene & Direction & Primer sequences $\left(5^{\prime}-3^{\prime}\right)$ \\
\hline \multirow[t]{2}{*}{ Catalase } & Forward & GCGAATGGAGAGGCAGTGTAC \\
\hline & Reverse & GAGTGACGTTGTCTTCATTAGCACTG \\
\hline \multirow[t]{2}{*}{ Cyclin D1 } & Forward & TTCGTGGCCTCTAAGATGAAGG \\
\hline & Reverse & TGAGCTTGTTCACCAGAAGCAG \\
\hline \multirow[t]{2}{*}{ Gapdh } & Forward & AGTGCCAGCCTCGTCTCATAG \\
\hline & Reverse & CCTTGACTGTGCCGTTGAACT \\
\hline \multirow[t]{2}{*}{ Gpx-1 } & Forward & GCTCACCCGCTCTTTACCTT \\
\hline & Reverse & GATGTCGATGGTGCGAAAGC \\
\hline \multirow[t]{2}{*}{$\|-1 \beta$} & Forward & AGGCTTCCTTGTGCAAGTGT \\
\hline & Reverse & TCCTGGGGAAGGCATTAGGA \\
\hline \multirow[t]{2}{*}{$\|-6$} & Forward & CACTTCACAAGTCGGAGGCT \\
\hline & Reverse & AGCACACTAGGTTTGCCGAG \\
\hline \multirow[t]{2}{*}{ Timp-1 } & Forward & ACAGCTTTCTGCAACTCGGA \\
\hline & Reverse & AGTTTGCAAGGGATGGCTGA \\
\hline \multirow[t]{2}{*}{ Timp-2 } & Forward & TGGGAACGTGCATTTTGCAG \\
\hline & Reverse & AAACACTGGTTGGAGGGCAA \\
\hline \multirow[t]{2}{*}{ Tnf-a } & Forward & CCAGACCCTCACACTCAGATCA \\
\hline & Reverse & TCCGCTTGGTGGTTTGCTA \\
\hline
\end{tabular}

Germany) with FastStart Essential DNA Green Master (Roche Diagnostics). GAPDH amplified in parallel served as the internal control.

\section{Clinical chemistry}

At sacrifice, the serum levels of ALT were measured using a standard clinical automatic analyzer (Type 7180; Hitachi, Tokyo, Japan).

\section{Hepatic lipid analysis}

Total lipids were extracted from approximately $200 \mathrm{mg}$ of liver tissue (frozen at sacrifice) for each rat, and the triglyceride levels were measured using the triglyceride E-test kit (Wako, Osaka, Japan) (Iwasa et al., 2010).

\section{Oxidative stress analysis}

Urinary 8-hydroxy-2'-deoxyguanosine (8-OHdG) levels were determined using an ELISA kit (NIKKEN SEIL, Shizuoka, Japan). Serum levels of hydroperoxide, one of the markers for oxidative stress, were determined using the derivatives of reactive oxygen metabolites (d-ROM) test (FREE Carpe Diem; Diacron s.r.l., Grosseto, Italy) (Kochi et al., 2013).

\section{Statistical analysis}

All data are expressed as mean $\pm \mathrm{SD}$, and one-way analysis of variance (ANOVA) was used for comparisons between groups. If ANOVA indicated significant differences, the Tukey-Kramer test for multiple comparisons was performed to compare the mean values among the groups. 
The differences were considered significant when the two-sided $P$ value was less than 0.05 . All analyses were conducted by the GraphPad InStat software, Version 3.05 (GraphPad Software; San Diego, CA, USA).

\begin{abstract}
Abbreviations
ALT: Alanine aminotransferase; ANOVA: Analysis of variance; Ctrl: Control; DEN: Diethylnitrosamine; d-ROM: Derivatives of reactive oxygen metabolites; EGCG: (-)-epigallocatechin-3-gallate; GAPDH: Glyceraldehyde-3-phosphate dehydrogenase; GPx: Glutathione peroxidase; GST-P: Glutathione Stransferase placental form; GTC: Green tea catechin; HCC: Hepatocellular carcinoma; HFD: High-fat diet; IL: Interleukin; NAFLD: Non-alcoholic fatty liver disease; NAS: NAFLD activity score; NASH: Non-alcoholic steatohepatitis; 8-OHdG: 8-hydroxy-2'-deoxyguanosine; RT-PCR: Reverse transcription-PCR; SD: Sprague-Dawley; TG: Triglyceride; TIMP: Tissue inhibitor of metalloproteinase; TNF: Tumor necrosis factor.
\end{abstract}

\section{Competing interest}

The authors declare that they have no competing interest.

\section{Authors' contributions}

TS made substantial contributions to conception and carried out the molecular biological studies. YS participated in the design of the study and carried out the molecular biological studies. MS participated in the design of the study and involved in drafting the manuscript and revising. TK carried out the molecular biological studies and made contributions to acquisition of data. TO made contributions to analysis of data. MK made contributions to analysis of data. MS involved in drafting the manuscript and revising. HT involved in drafting the manuscript and revising. TT carried out the pathological studies and performed the statistical analysis. HM made substantial contributions to analysis and interpretation of data and involved in drafting the manuscript and revising. All authors read and approved the final manuscript.

\section{Acknowledgments}

This work was supported in part by Grants-in-Aid from the Ministry of Education, Science, Sports and Culture of Japan (No. 22790638 and No.25460988), Grant-inAid for the 3rd Term Comprehensive 10-Year Strategy for Cancer Control from the Ministry of Health, Labor and Welfare of Japan, and Takeda Science Foundation.

\section{Author details}

${ }^{1}$ Department of Internal Medicine, Gifu University Graduate School of Medicine, Gifu, Japan. ${ }^{2}$ Department of Tumor Pathology, Gifu University Graduate School of Medicine, Gifu, Japan. ${ }^{3}$ Department of Internal Medicine/ Gastroenterology, Gifu University Graduate School of Medicine, 1-1 Yanagido, Gifu 501-1194, Japan.

Received: 26 October 2013 Accepted: 21 December 2013 Published: 27 December 2013

\section{References}

Adams LA, Lymp JF, St Sauver J, Sanderson SO, Lindor KD, Feldstein A, Angulo P (2005) The natural history of nonalcoholic fatty liver disease: a populationbased cohort study. Gastroenterology 129(1):113-121

Ando N, Shimizu M, Okuno M, Matsushima-Nishiwaki R, Tsurumi H, Tanaka T, Moriwaki $H$ (2007) Expression of retinoid $X$ receptor alpha is decreased in 3'-methyl-4-dimethylaminoazobenzene-induced hepatocellular carcinoma in rats. Oncol Rep 18(4):879-884

Ascha MS, Hanouneh IA, Lopez R, Tamimi TA, Feldstein AF, Zein NN (2010) The incidence and risk factors of hepatocellular carcinoma in patients with nonalcoholic steatohepatitis. Hepatology 51(6):1972-1978. doi:10.1002/hep.23527

Bettuzzi S, Brausi M, Rizzi F, Castagnetti G, Peracchia G, Corti A (2006) Chemoprevention of human prostate cancer by oral administration of green tea catechins in volunteers with high-grade prostate intraepithelial neoplasia: a preliminary report from a one-year proof-of-principle study. Cancer Res 66(2):1234-1240. doi:10.1158/0008-5472.CAN-05-1145
Chiang DJ, Pritchard MT, Nagy LE (2011) Obesity, diabetes mellitus, and liver fibrosis. Am J Physiol Gastrointest Liver Physiol 300(5):G697-G702. doi:10.1152/ajpgi.00426.2010

Cusi K (2012) Role of obesity and lipotoxicity in the development of nonalcoholic steatohepatitis: pathophysiology and clinical implications. Gastroenterology 142(4)::711-725. e716. doi:10.1053/j.gastro.2012.02.003

Day CP, James OF (1998) Steatohepatitis: a tale of two "hits"? Gastroenterology 114(4):842-845

Grove KA, Lambert JD (2010) Laboratory, epidemiological, and human intervention studies show that tea (Camellia sinensis) may be useful in the prevention of obesity. J Nutr 140(3):446-453. doi:10.3945/jn.109.115972

Hebbard L, George J (2011) Animal models of nonalcoholic fatty liver disease. Nat Rev Gastroenterol Hepatol 8(1):35-44. doi:10.1038/nrgastro.2010.191

Iwasa J, Shimizu M, Shiraki M, Shirakami Y, Sakai H, Terakura Y, Takai K, Tsurumi H, Tanaka T, Moriwaki H (2010) Dietary supplementation with branched-chain amino acids suppresses diethylnitrosamine-induced liver tumorigenesis in obese and diabetic C57BL/KsJ-db/db mice. Cancer Sci 101 (2):460-467. doi:10.1111/j.1349-7006.2009.01402.x

Kleiner DE, Brunt EM, Van Natta M, Behling C, Contos MJ, Cummings OW, Ferrell LD, Liu YC, Torbenson MS, Unalp-Arida A, Yeh M, McCullough AJ, Sanyal AJ (2005) Design and validation of a histological scoring system for nonalcoholic fatty liver disease. Hepatology 41(6):1313-1321. doi:10.1002/hep.20701

Kochi T, Shimizu M, Terakura D, Baba A, Ohno T, Kubota M, Shirakami Y, Tsurumi H, Tanaka T, Moriwaki H (2013) Non-alcoholic steatohepatitis and preneoplastic lesions develop in the liver of obese and hypertensive rats: suppressing effects of EGCG on the development of liver lesions. Cancer Lett. doi:10.1016/j.canlet.2013.08.031

Kuzu N, Bahcecioglu IH, Dagli AF, Ozercan IH, Ustundag B, Sahin K (2008) Epigallocatechin gallate attenuates experimental non-alcoholic steatohepatitis induced by high fat diet. J Gastroenterol Hepatol 23(8 Pt 2):e465-e470. doi:10.1111/j.1440-1746.2007.05052.x

Masterjohn C, Bruno RS (2012) Therapeutic potential of green tea in nonalcoholic fatty liver disease. Nutr Rev 70(1):41-56. doi:10.1111/j.1753-4887.2011.00440.x

Park EJ, Lee JH, Yu GY, He G, Ali SR, Holzer RG, Osterreicher CH, Takahashi H, Karin M (2010) Dietary and genetic obesity promote liver inflammation and tumorigenesis by enhancing IL-6 and TNF expression. Cell 140(2):197-208. doi:10.1016/j.cell.2009.12.052

Park HJ, DiNatale DA, Chung MY, Park YK, Lee JY, Koo SI, O'Connor M, Manautou JE, Bruno RS (2011) Green tea extract attenuates hepatic steatosis by decreasing adipose lipogenesis and enhancing hepatic antioxidant defenses in ob/ob mice. J Nutr Biochem 22(4):393-400. doi:10.1016/j.jnutbio.2010.03.009

Powell EE, Jonsson JR, Clouston AD (2005) Steatosis: co-factor in other liver diseases. Hepatology 42(1):5-13. doi:10.1002/hep.20750

Sanyal AJ, Campbell-Sargent C, Mirshahi F, Rizzo WB, Contos MJ, Sterling RK, Luketic VA, Shiffman ML, Clore JN (2001) Nonalcoholic steatohepatitis: association of insulin resistance and mitochondrial abnormalities. Gastroenterology 120(5):1183-1192. doi:10.1053/gast.2001.23256

Sanyal AJ, Chalasani N, Kowdley KV, McCullough A, Diehl AM, Bass NM, Neuschwander-Tetri BA, Lavine JE, Tonascia J, Unalp A, Van Natta M, Clark J, Brunt EM, Kleiner DE, Hoofnagle JH, Robuck PR (2010) Pioglitazone, vitamin E, or placebo for nonalcoholic steatohepatitis. N Engl J Med 362(18):1675-1685. doi:10.1056/NEJMoa0907929

Schattenberg JM, Galle PR (2010) Animal models of non-alcoholic steatohepatitis: of mice and man. Dig Dis 28(1):247-254. doi:10.1159/000282097

Shimizu M, Fukutomi Y, Ninomiya M, Nagura K, Kato T, Araki H, Suganuma M, Fujiki H, Moriwaki H (2008a) Green tea extracts for the prevention of metachronous colorectal adenomas: a pilot study. Cancer Epidemiol Biomarkers Prev 17(11):3020-3025. doi:10.1158/1055-9965.EPI-08-0528

Shimizu M, Shirakami Y, Sakai H, Adachi S, Hata K, Hirose Y, Tsurumi H, Tanaka T, Moriwaki H (2008b) (-)-Epigallocatechin gallate suppresses azoxymethaneinduced colonic premalignant lesions in male C57BL/KsJ-db/db mice. Cancer Prev Res (Phila) 1(4):298-304. doi:10.1158/1940-6207.CAPR-08-0045

Shimizu M, Sakai H, Shirakami Y, Iwasa J, Yasuda Y, Kubota M, Takai K, Tsurumi H, Tanaka T, Moriwaki H (2011a) Acyclic retinoid inhibits diethylnitrosamineinduced liver tumorigenesis in obese and diabetic C57BLKS/J-+(db)/+Lepr(db) mice. Cancer Prev Res (Phila) 4(1):128-136. doi:10.1158/1940-6207.CAPR-10-0163

Shimizu M, Sakai H, Shirakami Y, Yasuda Y, Kubota M, Terakura D, Baba A, Ohno T, Hara Y, Tanaka T, Moriwaki H (2011b) Preventive effects of $(-)$-epigallocatechin gallate on diethylnitrosamine-induced liver tumorigenesis in obese and diabetic C57BL/KsJ-db/db mice. Cancer Prev Res (Phila) 4(3):396-403. doi:10.1158/1940-6207.CAPR-10-0331 
Shimizu M, Yasuda Y, Sakai H, Kubota M, Terakura D, Baba A, Ohno T, Kochi T, Tsurumi H, Tanaka T, Moriwaki H (2011c) Pitavastatin suppresses diethylnitrosamine-induced liver preneoplasms in male C57BL/KsJ-db/db obese mice. BMC Cancer 11(1):281. doi:10.1186/1471-2407-11-281

Shimizu M, Kubota M, Tanaka T, Moriwaki H (2012) Nutraceutical approach for preventing obesity-related colorectal and liver carcinogenesis. Int J Mol Sci 13(1):579-595. doi:10.3390/ijms13010579

Shimizu M, Tanaka T, Moriwaki H (2013) Obesity and hepatocellular carcinoma: targeting obesity-related inflammation for chemoprevention of liver carcinogenesis. Semin Immunopathol 35(2):191-202. doi:10.1007/s00281-012-0336-6

Shirakami Y, Shimizu M, Tsurumi H, Hara Y, Tanaka T, Moriwaki H (2008) EGCG and Polyphenon $\mathrm{E}$ attenuate inflammation-related mouse colon carcinogenesis induced by AOM plus DDS. Mol Med Rep 1(3):355-361

Shirakami Y, Shimizu M, Adachi S, Sakai H, Nakagawa T, Yasuda Y, Tsurumi H, Hara Y, Moriwaki H (2009) (-)-Epigallocatechin gallate suppresses the growth of human hepatocellular carcinoma cells by inhibiting activation of the vascular endothelial growth factor-vascular endothelial growth factor receptor axis. Cancer Sci 100(10):1957-1962. doi:10.1111/j.1349-7006.2009.01241.x

Siegel AB, Zhu AX (2009) Metabolic syndrome and hepatocellular carcinoma: two growing epidemics with a potential link. Cancer 115(24):5651-5661. doi:10.1002/cncr.24687

Suzuki Y, Imai K, Takai K, Hanai T, Hayashi H, Naiki T, Nishigaki Y, Tomita E, Shimizu M, Moriwaki H (2013) Hepatocellular carcinoma patients with increased oxidative stress levels are prone to recurrence after curative treatment: a prospective case series study using the $d-$ ROM test. J Cancer Res Clin Oncol 139(5):845-852. doi:10.1007/s00432-013-1389-1

Terakura D, Shimizu M, Iwasa J, Baba A, Kochi T, Ohno T, Kubota M, Shirakami Y, Shiraki M, Takai K, Tsurumi H, Tanaka T, Moriwaki H (2012) Preventive effects of branched-chain amino acid supplementation on the spontaneous development of hepatic preneoplastic lesions in C57BL/KsJ-db/db obese mice. Carcinogenesis 33(12):2499-2506. doi:10.1093/carcin/bgs303

Thielecke F, Boschmann M (2009) The potential role of green tea catechins in the prevention of the metabolic syndrome - a review. Phytochemistry 70(1):11-24. doi:10.1016/j.phytochem.2008.11.011

Tsuda H, Fukushima S, Wanibuchi H, Morimura K, Nakae D, Imaida K, Tatematsu M, Hirose M, Wakabayashi K, Moore MA (2003) Value of GST-P positive preneoplastic hepatic foci in dose-response studies of hepatocarcinogenesis: evidence for practical thresholds with both genotoxic and nongenotoxic carcinogens. A review of recent work. Toxicol Pathol 31(1):80-86

Ueno T, Torimura T, Nakamura T, Sivakumar R, Nakayama H, Otabe S, Yuan X, Yamada K, Hashimoto O, Inoue K, Koga H, Sata M (2009) Epigallocatechin-3gallate improves nonalcoholic steatohepatitis model mice expressing nuclear sterol regulatory element binding protein-1c in adipose tissue. Int J Mol Med 24(1):17-22

Wang ZY, Agarwal R, Bickers DR, Mukhtar H (1991) Protection against ultraviolet B radiation-induced photocarcinogenesis in hairless mice by green tea polyphenols. Carcinogenesis 12(8):1527-1530

Wang Y, Ausman LM, Greenberg AS, Russell RM, Wang XD (2009) Nonalcoholic steatohepatitis induced by a high-fat diet promotes diethylnitrosamineinitiated early hepatocarcinogenesis in rats. Int J Cancer 124(3):540-546. doi:10.1002/ijc.23995

Wang Y, Ausman LM, Greenberg AS, Russell RM, Wang XD (2010) Dietary lycopene and tomato extract supplementations inhibit nonalcoholic steatohepatitis-promoted hepatocarcinogenesis in rats. Int J Cancer 126(8):1788-1796. doi:10.1002/ijc.24689

Yang S, Lin HZ, Hwang J, Chacko VP, Diehl AM (2001) Hepatic hyperplasia in noncirrhotic fatty livers: is obesity-related hepatic steatosis a premalignant condition? Cancer Res 61(13):5016-5023

Yang CS, Wang X, Lu G, Picinich SC (2009) Cancer prevention by tea: animal studies, molecular mechanisms and human relevance. Nat Rev Cancer 9(6):429-439. doi:10.1038/nrc2641

Zein CO, Yerian LM, Gogate P, Lopez R, Kirwan JP, Feldstein AE, McCullough AJ (2011) Pentoxifylline improves nonalcoholic steatohepatitis: a randomized placebo-controlled trial. Hepatology 54(5):1610-1619. doi:10.1002/hep.24544

doi:10.1186/2193-1801-2-690

Cite this article as: Sumi et al:: (-)-Epigallocatechin-3-gallate suppresses hepatic preneoplastic lesions developed in a novel rat model of non-alcoholic steatohepatitis. SpringerPlus 2013 2:690.

\section{Submit your manuscript to a SpringerOpen ${ }^{\circ}$ journal and benefit from:}

- Convenient online submission

- Rigorous peer review

- Immediate publication on acceptance

- Open access: articles freely available online

- High visibility within the field

- Retaining the copyright to your article

Submit your next manuscript at $\gg$ springeropen.com 